\title{
Automatic identification of edge chipping defects in high precision drilling of cemented carbide
}

\author{
Paolo Parenti*a ${ }^{*}$ Luca Pagani $^{\mathrm{b}}$, Massimiliano Annoni ${ }^{\mathrm{a}}$ \\ ${ }^{a}$ Department of Mechanical Engineering, Politecnico di Milano, via La Masa 1, 20156, Milan, Italy \\ ${ }^{b}$ EPSRC Future Metrology Hub, University of Huddersfield, Huddersfield, Queensgate, HD13DH, UK \\ *corresponding author: Email: paolo.parenti@polimi.it Tel: +3902 23998559
}

\begin{abstract}
Automatic visual inspection methods for product quality checking are spreading more and more in the present 4.0 manufacturing industry. This paper addresses the automatic inspection of edge integrity in accurate holes obtained by direct drilling of cemented carbide with innovative diamond coated tools. These revolutionary cutting tools, recently appeared on the market, can process extremely hard carbide in the sintered state, with massive increase of productivity with respect to standard methodologies like electrical discharge machining (EDM). However, due to the brittleness of the materials, the mechanical cutting process becomes critical and sensitive to tool breakage and workpiece defects generation. In particular, chipping of the hole edges represents one of the most important issues to monitor and take under control. A software procedure, that analyses high-resolution images taken from optical microscopes, was then developed for that aim. Image processing algorithms were designed and applied to enable the automatic extraction of the holes profile, thus permitting the identification and quantification of the leading edge damage in the radial direction. The proposed approach is fully automatic and is based on a profile segmentation that exploits an edge detection algorithm followed by a contour extraction method based on the solution of a partial differential equation. Dedicated metrics were specifically developed to evaluate the extracted profiles. The approach was validated with a factorial plane involving $1.6 \mathrm{~mm}$ diameter holes generated with different cutting parameters and tools on tungsten-carbide (WC) material. The technique resulted suitable for the aim, enabling the automatic characterisation of the defects generation phenomenon throughout the entire tools life. This moves a step toward the implementation of both in-line hole inspection procedures and advanced drilling process control.
\end{abstract}

Keywords: Image processing, Vision inspection, Edge defects, Hard metal, Drilling

\section{Introduction}

Hole inspection is particularly important in advanced drilling operation of difficult-to-cut materials. In these context, rapid identification, characterisation and classification of the process-induced defects become mandatory for enabling process optimisation and ensuring high quality and high precision production. Dedicated equipment and procedures that enable an automatic quality checking add value in the current Industry 4.0 era, by shortening the inspection time, by enabling the implementation of advanced process monitoring and control scheme and by supporting the development of unmanned production.

The newest and most productive technique to machine sintered tungsten-carbides (WC) at meso and micro scale is represented by the use of CVD (chemical vapour deposition) diamond coated tools [1]. Limited experience exists in the literature about the use of these tools with brittle workpiece materials, but one of the certain limitation is their sensitivity to chipping generation on the machined workpiece edges. The case of cemented WC drilling is particularly important because in these growing applications obtaining defect-free holes is mandatory for achieving comparable manufacturing quality in respect to the established machining technique, namely EDM (electrical discharge machining), and for improving the components fatigue life.

Hole border quality is usually evaluated in qualitative manners whilst the use of quantitative methods, especially in small holes, is less common because specific microscope tools and image processing techniques are required for the analysis.

Most of the image processing methodologies for defects identification have been developed in literature for addressing fiber reinforced composites machining in the medium diameter range $(2-10 \mathrm{~mm})$, to 
monitor the edge defects caused by fibers delamination (e.g. Giasin et al. [2] in GLARE material, Faraz et al. [3] in CFRP material and D'Orazio et al. [4] in carbon fiber reinforced plastic stacked with aluminium AA7075).

Moreover, only few attempts exist in literature about smaller scale holes. Aoyama et al. [5] analysed $1 \mathrm{~mm}$ diameter holes obtained by mechanical drilling in printed wiring board. Beruvides et al. [6] found a correlation between the holes border shape with the force signals in micro drilling of tungsten-copper alloy (holes diameter from $\phi 0.1-0.5 \mathrm{~mm}$ ).

In case of brittle materials drilling, the use of visual analysis methodologies that quantify the chipping phenomenon at the hole entrance is less studied and, to the best of our knowledge, no papers can be found about sintered carbides mechanical drilling, despite the many industrial applications that involve this type of material. Not only an automatic characterisation of the chipping phenomenon is useful for cutting monitoring purposes and part qualification but it may also supports the development of theoretical frameworks, involving fracture mechanics and cutting induced behaviour ( $\operatorname{Lv}$ et al. [7], Wang et al. [8]) that help in avoiding the chipping generation. One of the few attempts to generalise and automatise the border damage identification was carried out in De Albuquerque et al. [9] where the damaged area around the holes were computed - starting from radiographic images - by training a neural network that segmented the input images. Despite that, it can be claimed that automatic identification, classification and clustering of border defects is not covered in literature.

Going into more details of the imaging algorithms and data extraction the literature shows that many possibilities are available but their applicability is strictly connected to the image characteristics. Advanced algorithms used to detect an object can be based on the image edges on the solution of a partial differential equation (PDE). Edge based methods, such as the Canny edge detection [10], compute the gradient of the image to find the edges, the object is then segmented connecting the edges. Due to the high number of edges found the connection operation, required to find the desired object, is often a difficult task. PDE based method, such as the geodesic active contour [11] or the variant without edges [12], solve a PDE in order to maximise a functional. Starting from a closed profile, it evolves according to some predefined PDE. PDE based methods are usually slower compared the to edge based ones, but they can produce a better segmentation. Due to the complexity of the analysed images and to the robustness required by the algorithm a PDE based method, proposed by Chan and Vese [12], is used in the present work.

This literature analysis shows that some drawbacks and limitations exist in the current solutions for hole border monitoring (i) manual interventions is usually prescribed to setup the algorithms (ii) commercial software with built-in generic routines are generally used to accomplishing border identification tasks, without full control of the imaging algorithms (iii) robustness and extension of the methodology to other measurement instruments, materials and application cases were not studied nor discussed (iv) no specific metrics have been proposed for analysing the case of brittle material edge damages $(v)$ the case of high precision drilled holes with small diameter, less than $2 \mathrm{~mm}$, is only slightly covered (vi) the case of small drilling with CVD diamond coated tools on cemented carbides has not been studied yet.

The present work studies a procedure for performing automatic edge chipping identification in high precision mechanical drilling of sintered carbide.

The novelty proposed by the present work is framed in two main areas namely: (i) the vision monitoring algorithms and (ii) the advanced manufacturing methods. On one side, the proposed approach for holes inspection in meso/micro drilling is fully automatic, then possessing high inherent impact for the industry 4.0 world. The algorithm is tested with images obtained from a focus variation microscope, therefore opening the possibility to implement in-process scanning of the hole quality, potentially integrating additional metrology operations. From the advanced manufacturing point of view, the methodology is applied to high precision drilling of sintered carbides with diamond coated tools that is a ground-breaking manufacturing technique with high potential and perspective impact in industry. The presented experimental results therefore represent a first scientific investigation about the obtainable drilling quality, in terms of process capability and hole accuracy data, that can be used as benchmark for comparing this advanced manufacturing techniques with other and more established ones. The paper is organised as follow: in Section 2 the experimental setup is described, in Section 3 the principle of the chipping defect formation is briefly recalled, in Section 4 the segmentation process is described, in Section 5 the metric used to characterise the manufacturing defect are presented, in Section 6 the results of the analysis of the experiments is discussed, in Section 7 a sensitivity analysis is performed and in Section 8 conclusions and future developments are given. 


\section{Equipment and Materials}

\subsection{Drilling of sintered carbides}

The drilling operations were conducted on an ultra-high precision milling centre KERN ${ }^{\circledR}$ Evo (resolution $0.1 \mu \mathrm{m}$, positioning accuracy $\pm 1 \mu \mathrm{m}$ ) equipped with a CNC control from HEIDENHAIN ${ }^{\circledR}$ (iTNC 530). The tools were carbide twist drills with diameter of $1.6 \mathrm{~mm}$ (UDCMX 2160-100) from Union Tool ${ }^{\circledR}$, characterised by CVD diamond coating technologies that make them suitable for machining cemented carbides with extreme hardness. Holes depth was fixed to $8 \mathrm{~mm}$, producing dead end holes. Tools were preset by using Marposs Visual Tool Setter ${ }^{\circledR}$ that allowed the measurement of the flying diameter and run-out of each tool (TIR-Total Indicator Reading) - less than $3 \mu \mathrm{m}$ for all of them. Machining was conducted with peck drilling strategy - as suggested by the tool builder - with pressurised chilled air as cooling method (Cold Gun system from Exair ${ }^{\circledR}$ ). The holes were obtained in a circular specimen $35 \mathrm{~mm} \times 15 \mathrm{~mm}(\mathrm{D} \times \mathrm{H})$ made in a medium grade sintered tungsten-carbide with $10 \%$ of cobalt binder (WC-Co 10w\%) produced by F.I.L.M.S. Spa and characterised by a hardness of $1550 \mathrm{HV}$ with initial roughness - after grinding finish - of around $0.8 \mu \mathrm{m}$. The overall experimental setup and analysis procedure is depicted in Figure 1.
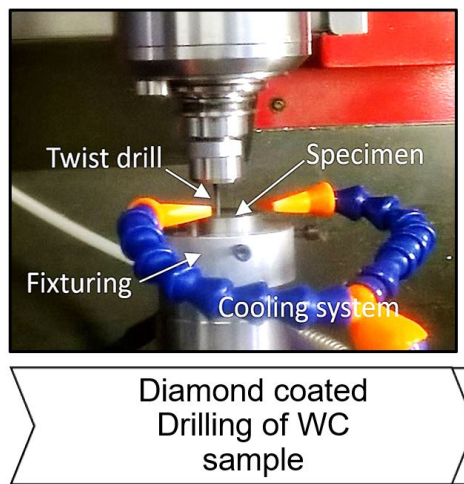

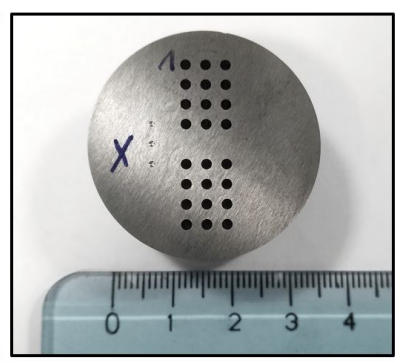

WC sample
With 24 Holes
$\Phi=1.6 \mathrm{~mm}$

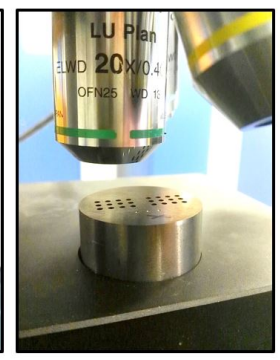

3D

Microscope Acquisition

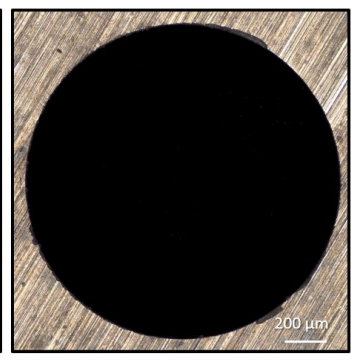

24-bit Color image (5000x5000 pixel)

Figure 1: Setup and Experiments Chain

\subsection{Design of Experiments and cutting conditions}

A factorial plane was conducted with a $2^{3}$ design, three replicates and a block factor i.e. the tool. Three identical tool units were adopted in way that each tool machined a total number of eight holes for a total amount of drilling length equals to $64 \mathrm{~mm}$, very much lower that the declared wear limit by the tool manufacturer. The three factors were cutting speed (12 and $\left.18 \frac{\mathrm{m}}{\mathrm{min}}\right)$, feed rate (48 and $\left.72 \frac{\mathrm{m}}{\min }\right)$ and peck-step height $(0.08$ and $0.12 \mathrm{~mm})$. In peck drilling, the tool intermittently disengages from the cutting by alternating axial movements in feed and opposite direction. The peck step height represents the axial length that the tool covers into the cutting at the prescribed feed rate. The drilling tests were fully randomised both spatially and in respect to run order.

\subsection{Microscope Measurements}

The samples were inspected with Alicona ${ }^{\circledR}$ Infinite Focus G4 Microscope with different objectives (from $5 \mathrm{x}$ to $20 \mathrm{x}$ ). Light was supplied by the coaxial illumination ring. Vertical resolution was fixed to $0.4 \mu \mathrm{m}, 0.3 \mu \mathrm{m}$ and $0.2 \mu \mathrm{m}$, for the $5 \mathrm{x}, 10 \mathrm{x}$ and $20 \mathrm{x}$ lens respectively. Lateral resolution instead was fixed to $3 \mu \mathrm{m}, 2 \mu \mathrm{m}$ and $1 \mu \mathrm{m}$, respectively, with no decimation. The microscope was able to capture both height-map and colour images of the upper surface of the holes by stitching a number of $5 \times 4$ patches using the 20x objective. Due to the major contrast guaranteed by the colours map it was decided to use these images for the analysis. Total scanning depth was adjusted case by case but never over-passed the $80 \mathrm{\mu m}$ range. The images were exported in bitmap format with 24-bit colour depth with an approximate size of about $5000 \times 5000$ pixels each. To evaluate the effect of different objective the images were taken with the $5 \mathrm{x}$ and $10 \mathrm{x}$ objective too, they will be analysed in Section 7.2. Table 1 summarises the measurement parameters for each of the objective used. 


\begin{tabular}{|c|c|c|c|c|c|}
\hline Lens & $\begin{array}{c}\text { Lateral } \\
\text { resolution }(\mu \mathrm{m})\end{array}$ & $\begin{array}{c}\text { Vertical } \\
\text { resolution }(\mu \mathrm{m})\end{array}$ & $\begin{array}{l}\text { Stitched } \\
\text { images }\end{array}$ & $\begin{array}{c}\text { Exposure }(\mu s) / \\
\text { Contrast }\end{array}$ & $\begin{array}{l}\text { RGB image } \\
\text { resolution }\end{array}$ \\
\hline $20 \mathrm{x}$ & 0.324 & 2.935 & $5 \times 4$ & $136 / 0.6$ & $5904 \times 5362$ \\
\hline $10 \mathrm{x}$ & 0.443 & 3.914 & $3 \times 2$ & $122 / 0.6$ & $3050 \times 3298$ \\
\hline $5 \mathrm{x}$ & 1.788 & 7.828 & $2 \times 2$ & $115 / 0.6$ & $1628 \times 1236$ \\
\hline
\end{tabular}

Table 1: Measurement parameters of the focus variation microscope

\section{Chipping Defect}

The indisputable goal of drilling is producing holes with good geometrical and dimensional quality, smooth internal surfaces and defect free edges. This latter aspect is critical in hard metal drilling due to the material brittleness and the associated tendency to generate border fractures. In blind holes, the attention can be given to the entrance edge that is subjected to chipping defects (Figure 2). Entrance chipping is the results of the collapse of the leading edge portions - generated by the material stresses induced by the action of the drilling tool. Many causes associated to cutting process could play a role in the chipping onset as the tool/cutters geometries and misalignment [13] e.g. run-out, forced or self-induced vibration (axial/torsional chatter). At the same time, material characteristics could be extremely important with its anisotropic behaviour, internal stresses and internal local defects (pores, inclusions etc.). In any case, chipping reveals itself with a certain amount of radial and axial extension, which are typically coupled depending on the material and induced stress characteristics. Among these two directions, when the holes are deep, the attention is usually put on radial chipping during the quality controls inspections.
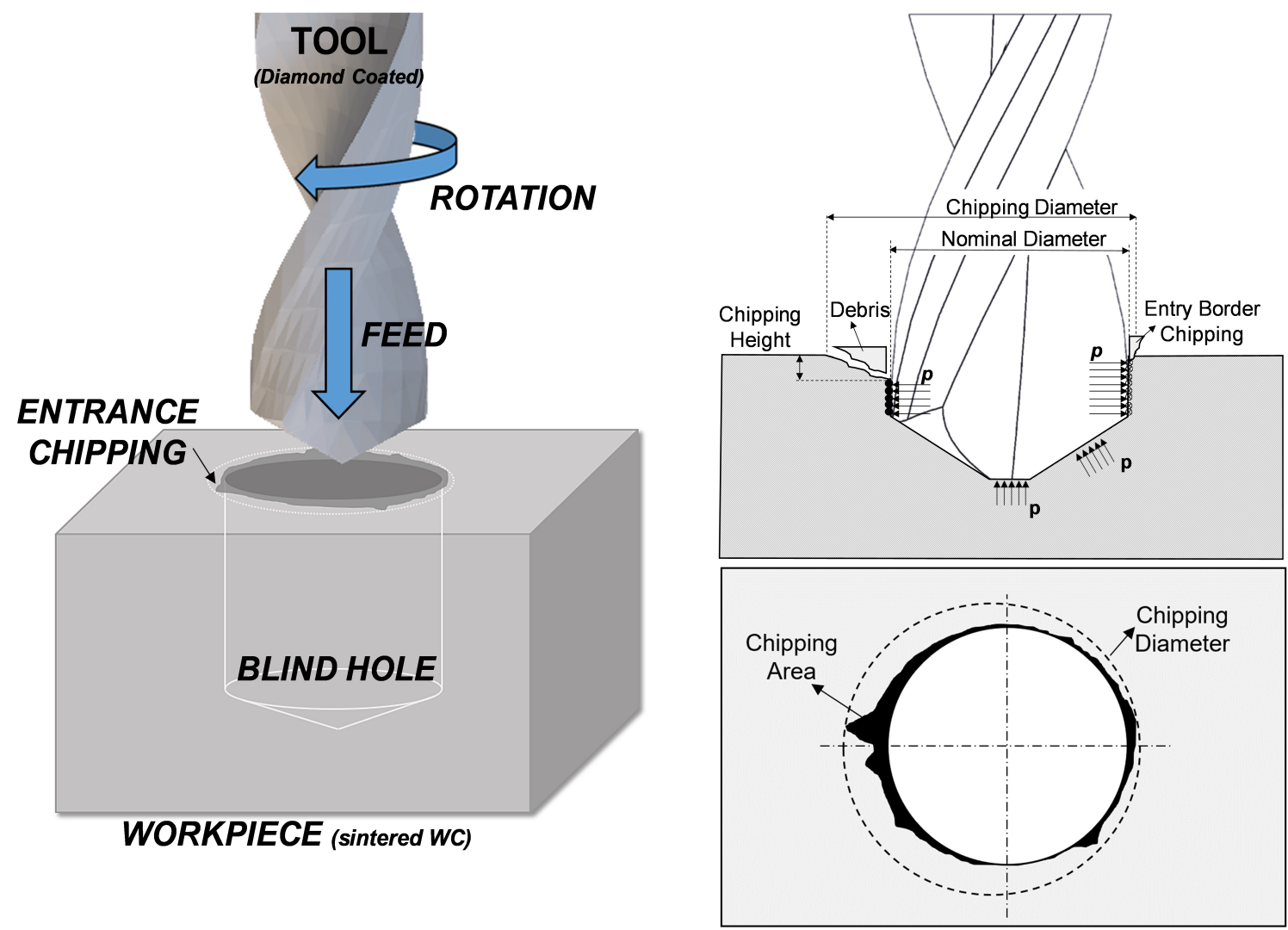

Figure 2: Defect generation in drilling

\subsection{Chipping Morphology}

In the analysed case, consisting in the Tungsten-Cobalt samples machined trough diamond coated tools, the border breakage exposes a typical brittle fracture zone which can be observed in Figure 3 whose radial extension is irregular and apparently random shaped. The radial deviation to the nominal profile - i.e. a perfect circle - and to the actual profile - i.e. the profile of the holes below the chipping 
height - can be determined by a normal upper view of the hole. Due to the small hole diameters and the limited extension of the defect, chipping can be analysed only trough microscope imaging analysis.

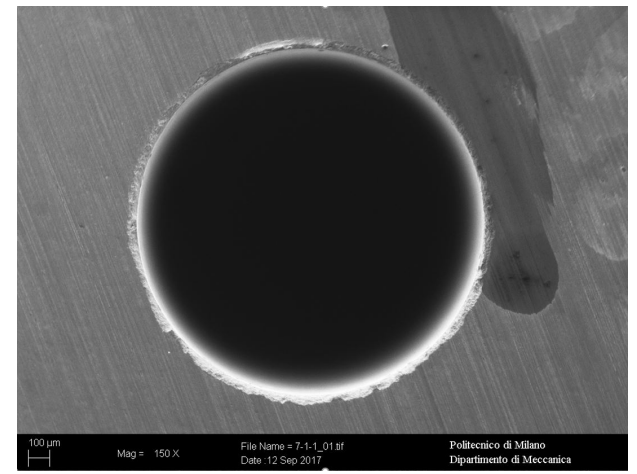

(a) Zoom at $150 \mathrm{x}$

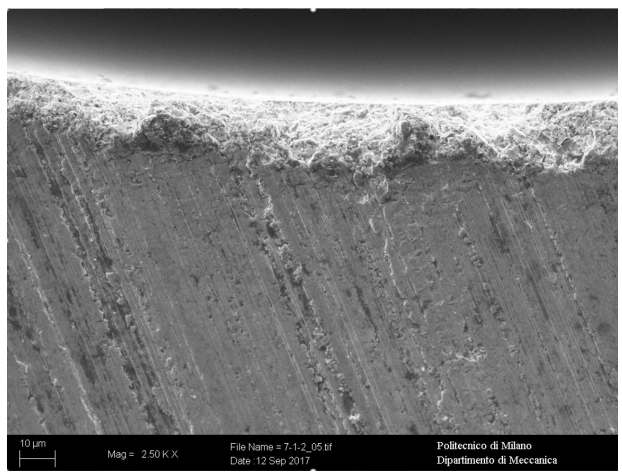

(b) Zoom at $2500 \mathrm{x}$

Figure 3: Scanning Electron Microscope (SEM) Images

\section{Image Processing Methodology}

The software methodology was applied on the obtained images and implemented in MATLAB ${ }^{\circledR}$. In order to compute the amount of radial chipping, the edges of the hole have to be extracted. In this Section, the procedures followed to segment the hole profile, using the active contours without edges algorithm proposed by Chan and Vese [12], and to obtain the chipping quality indices, described in Section 5, are investigated. The profile segmentation can be achieved with the following steps (summarised in the flowchart in Figure 4):

1. Apply edge detection filter;

2. Extract the contour of the hole;

3. Find a good starting point for the Chan and Vese algorithm [12];

4. Extract the contour with the Chan and Vese algorithm.

The first step of the segmentation process corresponds in finding a good starting point for the Chan and Vese algorithm. The RGB image is first converted to a grey level scale, then the Sobel method is applied to compute the gradient to enhance the edges without applying any pre-processing. After applying the edge detection algorithm using the automatic threshold implemented in MATLAB ${ }^{\circledR}$, i.e. thres $=\sqrt{\frac{4}{n_{x} \cdot n_{y}} \sum_{x} \sum_{y} I_{x}^{2}(x, y)+I_{y}^{2}(x, y)}$ (where $I_{\bullet}(x, y)$ is the partial derivative of the image in the - direction and $n_{x} \cdot n_{y}$ is the total number of pixels of the image), it is not possible to detect the edge of the hole (see Figure 5a). The image is dilated using the union of vertical, horizontal and diagonal structuring element of size 10 pixel each in order to connect the edges, then the longest contour at a grey level corresponding to 0.5 is extracted (Figure 5b). The maximum inscribed circle computed using the maximum distance transform, implemented in MATLAB ${ }^{\circledR}$ [14], is used as the starting point for the Chan and Vese method.

The aim of the algorithm proposed by Chan and Vese [12] is to divide an object from the background of an image, it can be then used to find the edge of the manufactured hole. Starting from an initial contour $C$ of the object, the segmentation is performed minimising the functional

$$
\begin{aligned}
F\left(c_{1}, c_{2}, C\right)= & \lambda_{1} \int_{\text {inside }(C)}\left|u(x, y)-c_{1}\right|^{2} d x d y \\
& +\lambda_{2} \int_{\text {outside }(C)}\left|u(x, y)-c_{2}\right|^{2} d x d y
\end{aligned}
$$

where $c_{1}\left(c_{2}\right)$ is the average value of $u(x, y)$ inside (outside) $C, \lambda_{1}$ and $\lambda_{2}$ are two positive or null constant that have to be set. The values $\lambda_{1}$ and $\lambda_{2}$ balance the contribution of the values inside and outside the the contour in the minimisation of the functional. The minimisation problem is then solved using the level set method [15]. Let $\phi(x, y)$ be a signed distance function, i.e. a function that measures the distance between a point $(x, y)$ and $C$, that is negative inside and positive outside the boundary. The contour of the hole is represented by the implicit function $\phi(x, y)=0$ that has to be computed. In order to minimise the functional a starting signed function has to be provided, then 


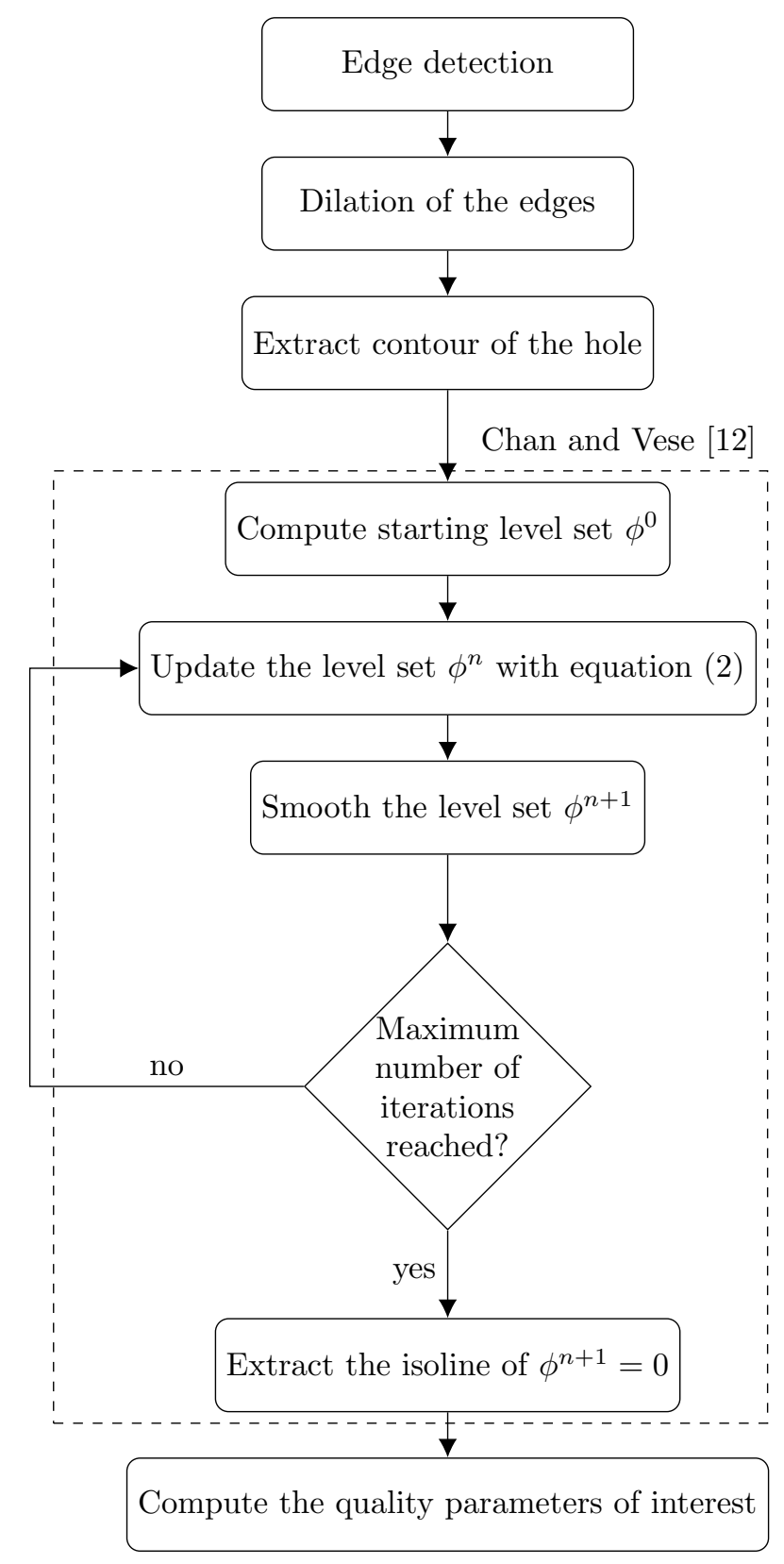

Figure 4: Flowchart

a numerical method can be applied to solve the segmentation problem using the following update equation

$$
\phi^{n+1}(x, y)=\phi^{n}(x, y)+\Delta t\left[\lambda_{2}\left(u(x, y)-c_{2}\right)^{2}-\lambda_{1}\left(u(x, y)-c_{1}\right)^{2}\right]
$$

where $\phi^{n}(x, y)$ is the signed distance function at the $n$-th step and $\Delta t$ is the time step of the numerical integration. The time step defines the evolution speed of the contour, with $\lambda_{1}$ and $\lambda_{2}$ set, a time step to small does not allow the evolution of the contour, while a time step to high does not allow the correct determination of the contour details. Due to the definition of a signed distance function, $c_{1}\left(c_{2}\right)$ can be easily computed averaging all the values of the image with $\phi(x, y)$ smaller or equal (greater) than zero. For each step of the algorithm a Laplace smoothing of the level set is performed, this further step is useful to avoid that the algorithm evolves in the surface grooves, generated by grinding the sample surface right before drilling, Figure 7. 


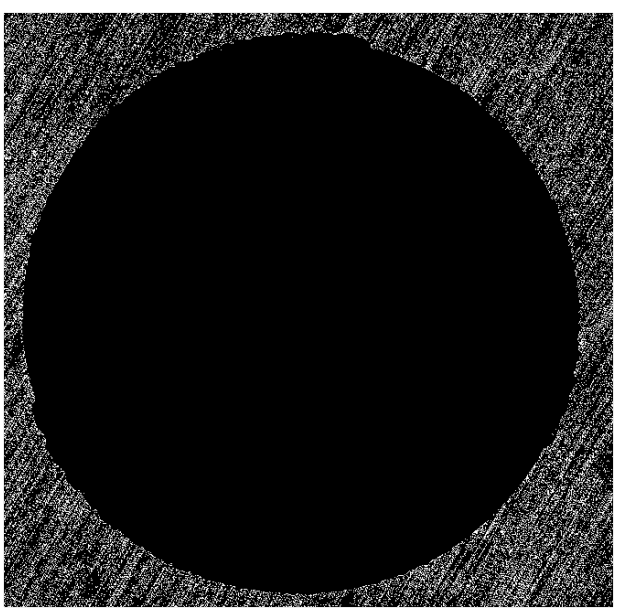

(a) Image after the Sobel operator

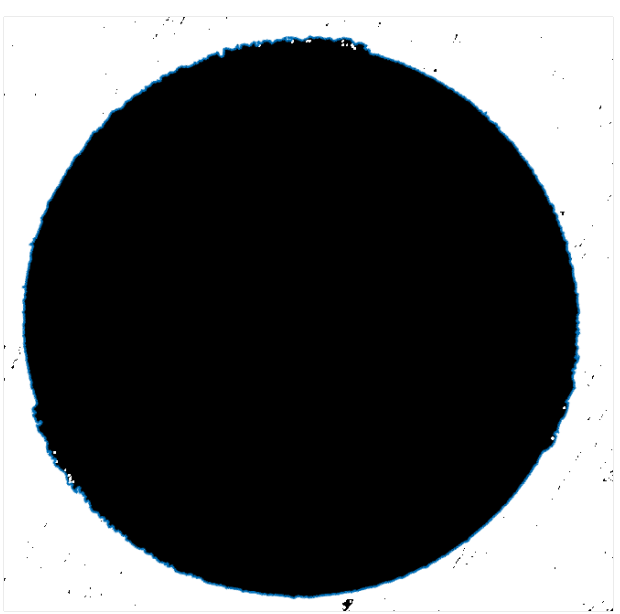

(b) Image after the dilation step

Figure 5: Steps to compute the profile

After applying the algorithm, setting a number of iterations equal to 20 with a time step equal to 0.5 , the segmented profile is obtained, as shown in Figure 6. Optimal tuning of $\lambda_{1}$ and $\lambda_{2}$ was for both a value of 0.004 , while smoothing weight for the Laplace algorithm was set to 50 . These values demonstrated for all the analysed images good performance by avoiding the grinding grooves tracking, previously discussed. It must be noted that big variations of initial surface roughness of the ground samples is not expected in real production environments thus alleviating the risk associated to this manual parameters tuning.

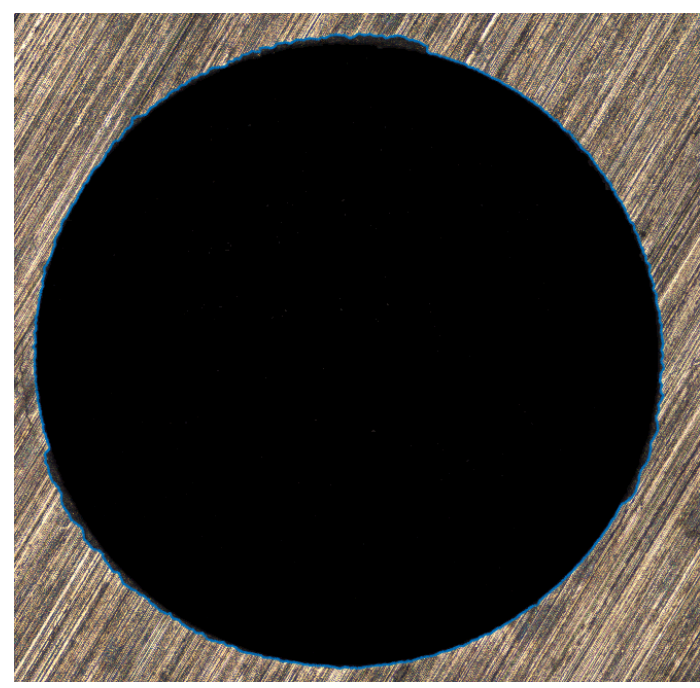

Figure 6: Segmented profile of the hole number 12

It should be noted that the smoothing weight is chosen as much greater than $\lambda_{1}$ and $\lambda_{2}$, to avoid the evolving profile to go into the grooves of the grinding operation. To show the effect of the smoothing weight, in Figure 7 two magnifications of the segmented profile with two different values are reported. It is possible to observe that the profile with the greatest weight is smoother, with the possibility to lose some small details, but the profile does not evolve wrongly by producing inexact segmentation. 


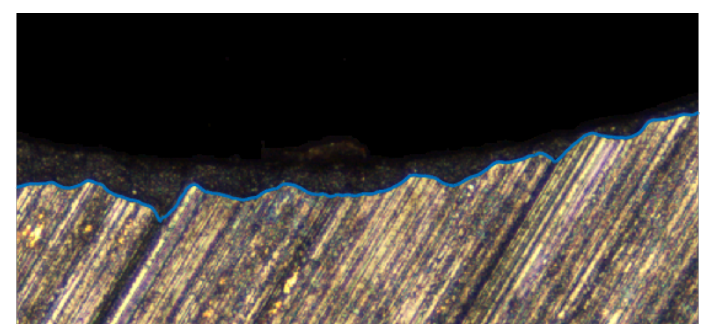

(a) Smoothing weight equal to 50

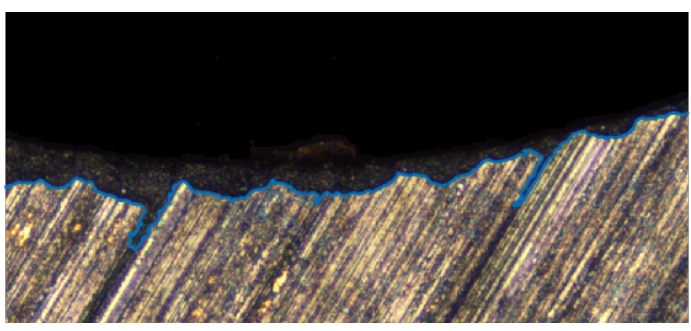

(b) Smoothing weight equal to 1

Figure 7: Effect of the smoothing factor in the edge segmentation

\section{Metric for evaluating chipping defect}

Different synthetic indicators can be used to assess the damage level at the hole edges. In most of the literature, due to the difficulty in measuring the damaged area standard optical microscope, the standard is to adopt both dimensional and a-dimensional 1-D indicators such as the maximum damaged diameter or the ratio between this latter and the nominal diameter [16]. Only when areal measurements are available, damaged 2-D coefficients - as the ratio between damaged area and nominal area of the hole - can be calculated allowing more extensive and robust accuracy error characterisation. Starting from this point, Davim et al. [17] proposed an adjusted damage factor (Fda) that have been extracted in delaminated fiber reinforced plastic holes (FRP) using digital analysis.

In the presented case of carbide drilling, after computing the maximum inscribed circle, the profile is first converted into polar coordinates. After the conversions of the extracted points in polar coordinates, using as reference centre the one of the maximum inscribed circle, the hole profiles were analysed as discussed in the following section.

The computed indices are the radius of the maximum inscribed circle $\left(\rho_{\min }\right)$, the radius of the circle that contains all the points with the centre coinciding with the maximum inscribed circle $\left(\rho_{\max }\right)$ and the edge chipping area, as following:

$$
A=\int_{0}^{2 \pi} \frac{\rho^{2}(\theta)-\rho_{\min }^{2}}{2} d \theta
$$

where $\rho(\theta)$ is the radius hole profile; the trapezoidal rule was used to compute the defect area.

A further index was computed to characterise the profiles with one or more lobes (see Figure 9). The proposed index is the kurtosis of the amplitude of the discrete Fourier transform (DFT), i.e. the spatial spectrum, of the profile. Fast Fourier transform (FFT) algorithm is applied for the computation of the spectrum using a FlatTop windowing and an input data size equal to 10000 points (angular resolution of 0.036 degree).

Once the metric for quantifying the edge chipping is defined and the measurement indicators are obtained, a further challenge consists in associating to these number the measurement uncertainty. A more accurate reference to compare and validate the results would be therefore needed. However, in this manuscript this aspect is not treated and it is left as a future development step. Moreover, in this analysis the obtained indicators are mainly used in relative way to assess differences due to different process conditions, making the lack of uncertainty evaluation less relevant.

\section{Results and Discussion}

In this paragraph, the data extracted by image processing are analysed. First, chipping morphology is discussed followed by the analysis of variance (ANOVA), conducted to assess the effect of the cutting parameters on the chipping indicators.

\subsection{Chipping profiles identification}

The identification worked well for all the 24 analysed holes and all the profiles were extracted for quantitative evaluation as illustrated in Figure 8. At a first look, much variation can be observed in the maximum radial deviation and on the profile shape. The maximum detected chipping width remains, however, below $60 \mu \mathrm{m}$. 


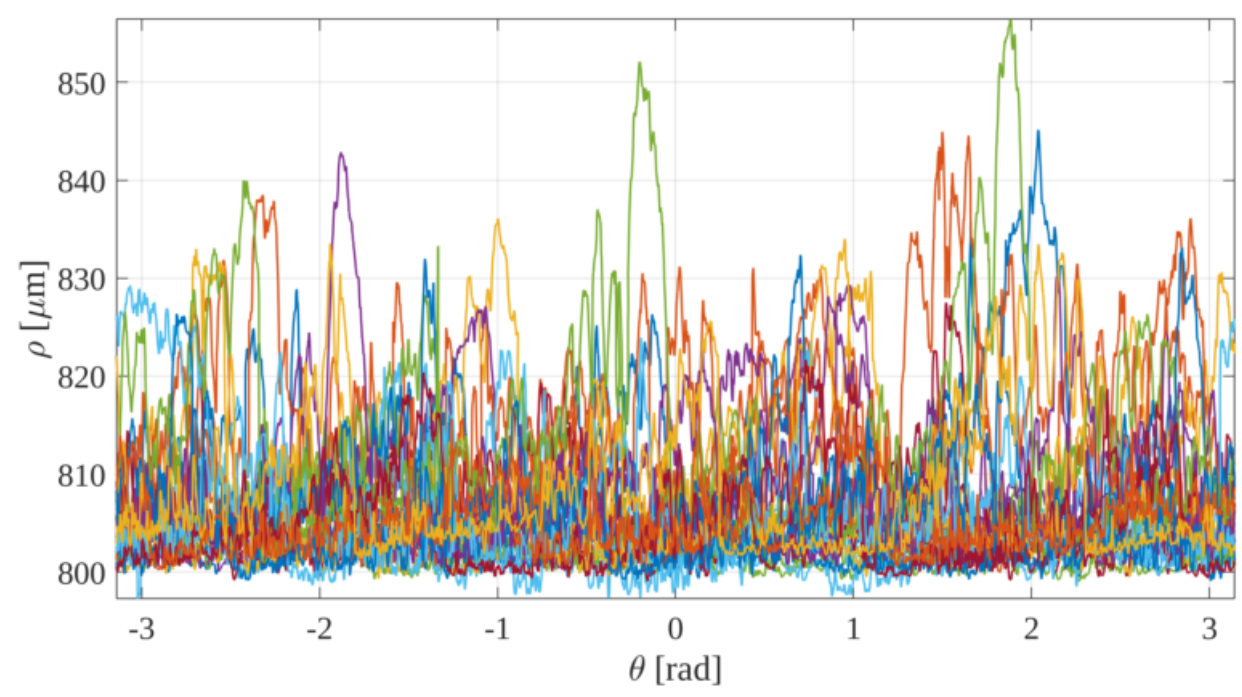

Figure 8: Radius of the segmented profiles (all 24 holes), plotted in polar coordinates

\subsubsection{Chipping morphology}

Chipping morphology ranges between random shaped to lobed one. In general, no particular "structures" can be observed in the profiles but some of the holes show the presence of lobes around the border circumference. The angular wavelength characterising these lobes is detected around $2 / 3 \pi$ range, around 3 lobes in the profile. Lobes look with similar shape among the holes, always showing a visible non-linear tendency. The kurtosis of the profiles DFT allows a good classification of this lobing tendency, as showed in Figure 10a. Holes \#3, \#12, \#19 and \#24 clearly emerged to belong to the lobed category.
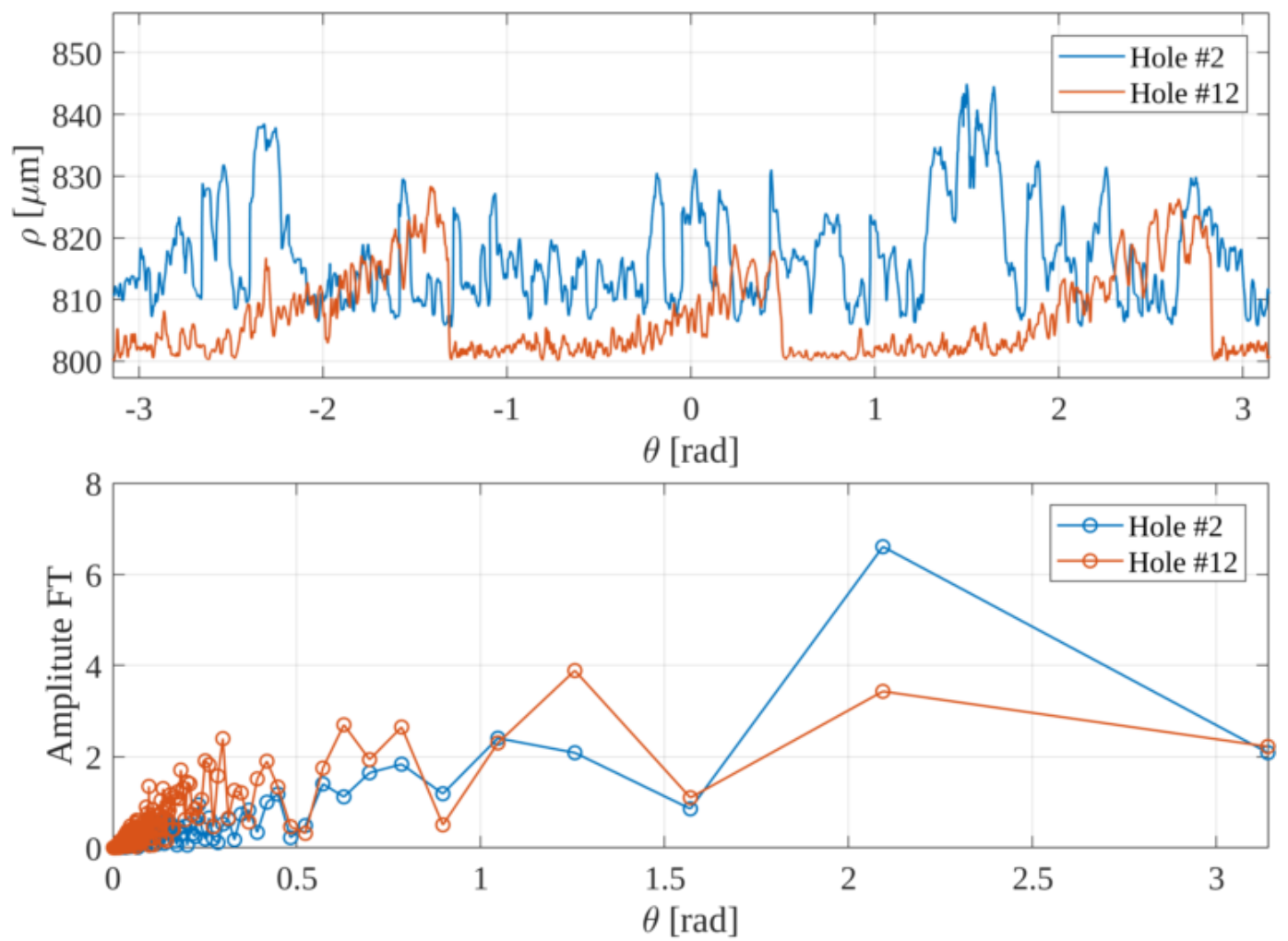

Figure 9: Profiles of Hole \#2 and \#12, profile in polar coordinates (up), DFT amplitude (bottom) 


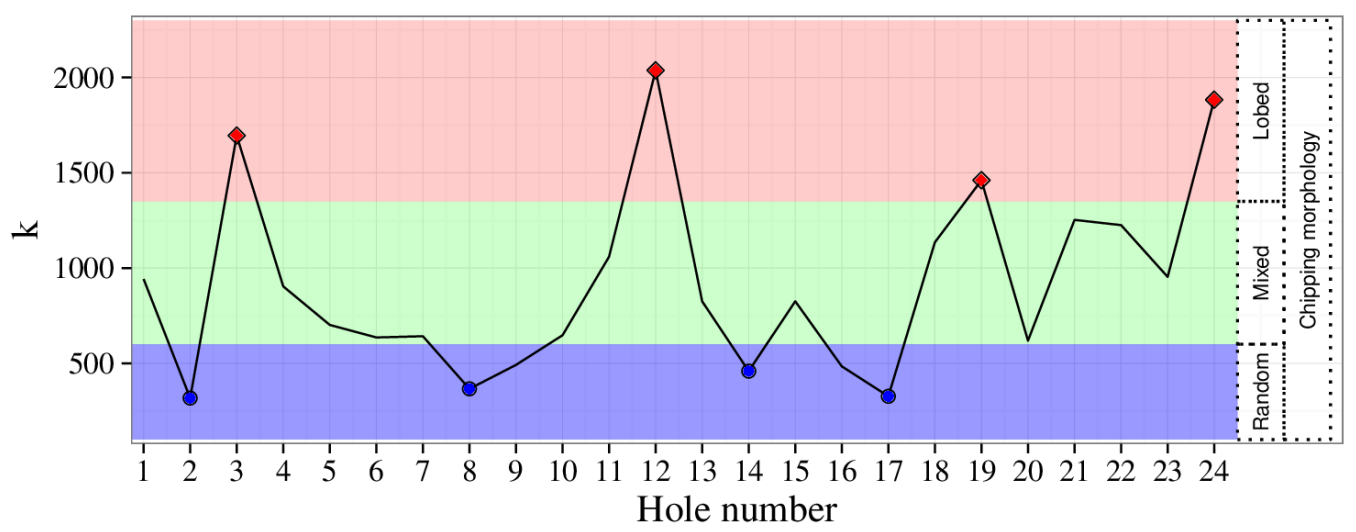

(a) Kurtosis (k) of the DFT amplitude of the profiles
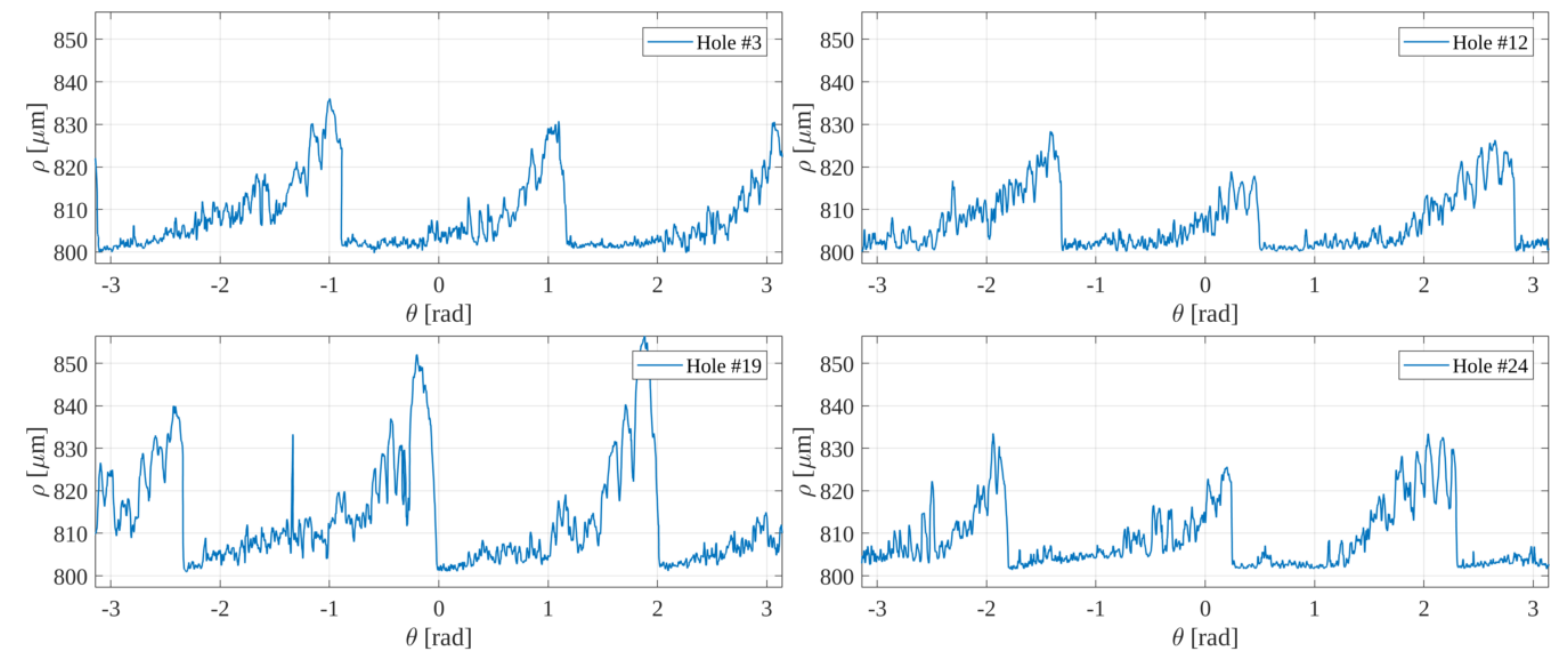

(b) Profiles of the holes \#3, \#12, \#19, \#24

Figure 10: Lobing classification of the holes profiles trough thresholding of the spatial spectrum kurtosis

\subsubsection{ANOVA on the cutting parameters}

The effect of the process parameters, $V c$, Feed and peck-step height, is analysed using the aforementioned indices describing the holes quality. Figure 11 shows the computed indices as a function of the process parameters along with the mean response and the $95 \%$ confidence interval on the mean. An ANOVA was then performed to investigate whether a factor has an effect on the final quality of the hole. As it is possible to observe there are some holes causing high variability in the responses, but the hypothesis on residuals of the ANOVA models were respected. The unusual observations correspond to two holes manufactured with the third tool in sequence.

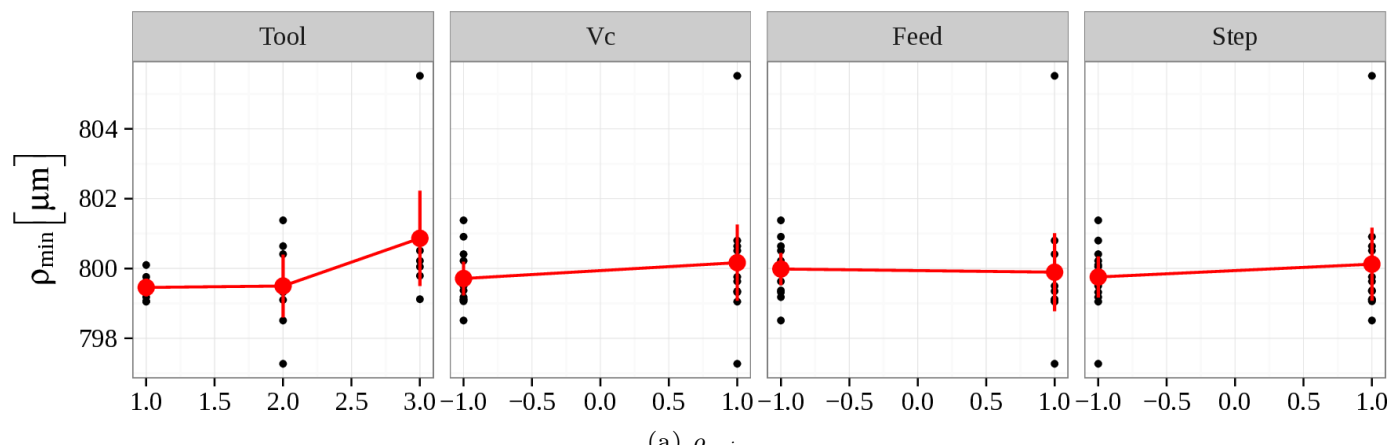




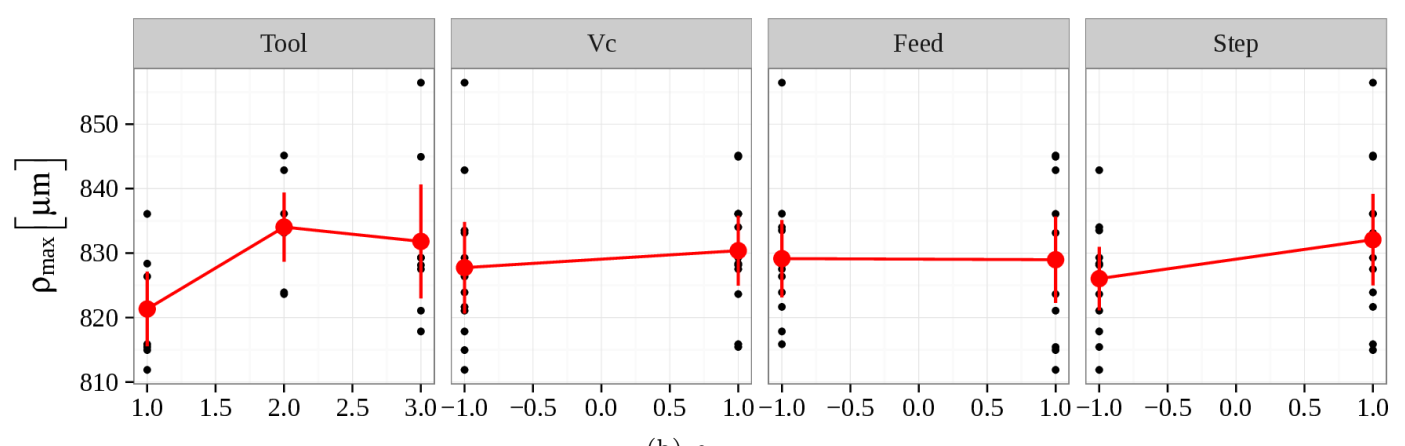

(b) $\rho_{\max }$

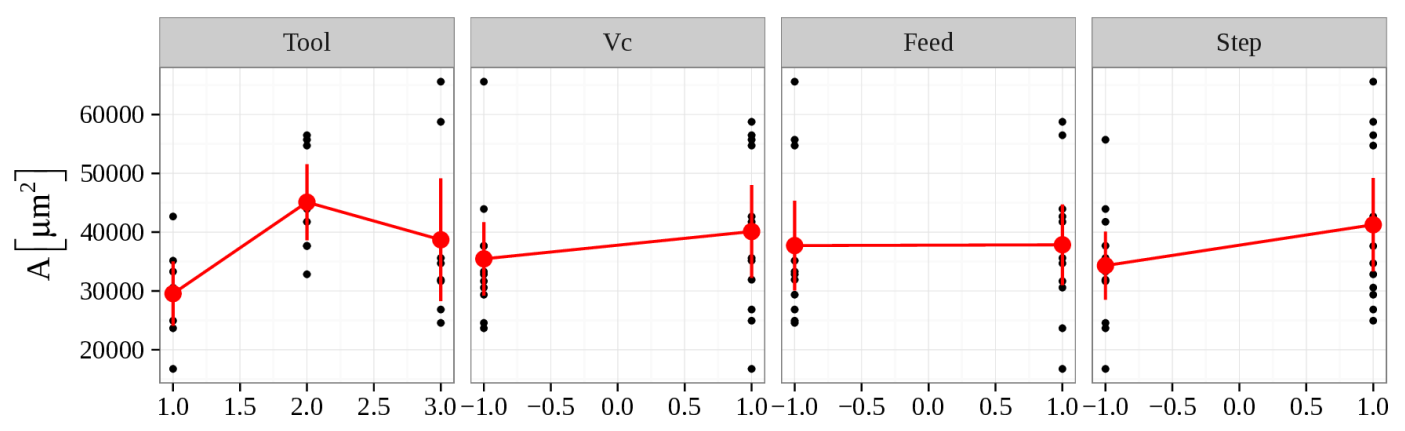

(c) $A$
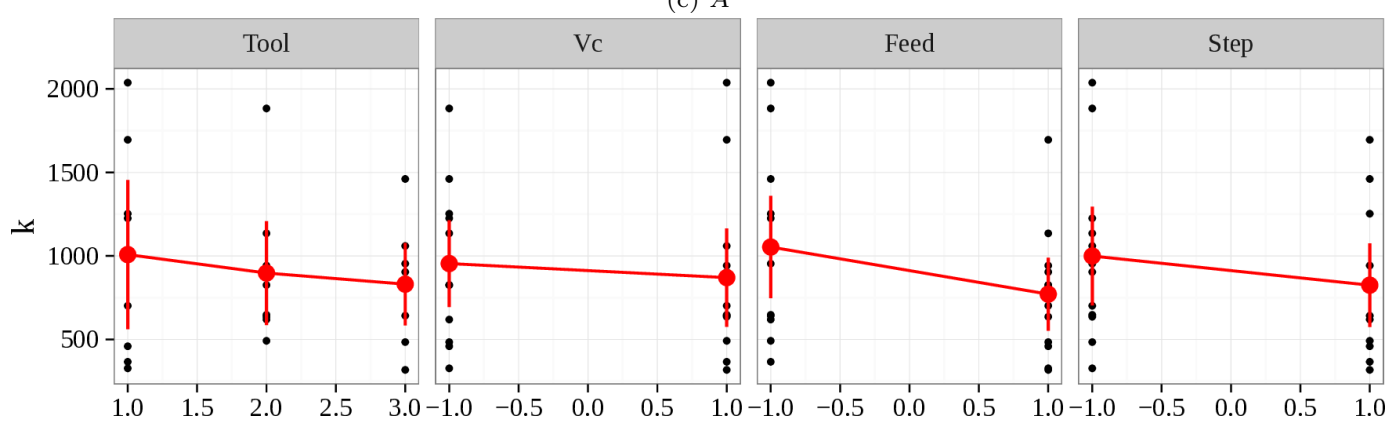

(d) $k$

Figure 11: Main effect plots of the analysed factors

From the p-values of the ANOVA tables (see Table 2), it is possible to conclude that the tool had an effect on both $\rho_{\max }$ and on the area of the defect, while the effect of the parameters on $\rho_{\min }$ is masked by the variability of the process. None of the analysed factor has any effect on the kurtosis of the DFT amplitude.

\begin{tabular}{l|c|c|c|c}
\hline \multicolumn{1}{c}{ Source } & $\rho_{\min }$ & $\rho_{\max }$ & $A$ & $k$ \\
\hline Block (Tool) & 0.101 & 0.036 & 0.031 & 0.759 \\
Vc & 0.433 & 0.499 & 0.294 & 0.659 \\
Feed & 0.874 & 0.965 & 0.976 & 0.174 \\
Step & 0.524 & 0.134 & 0.125 & 0.413 \\
Vc*Feed & 0.554 & 0.281 & 0.405 & 0.175 \\
Vc*Step & 0.070 & 0.653 & 0.823 & 0.689 \\
Feed*Step & 0.407 & 0.329 & 0.331 & 0.114 \\
Vc*Feed*Step $^{*}$ & 0.342 & 0.064 & 0.052 & 0.392 \\
\hline
\end{tabular}

Table 2: P-values ANOVA models

Figure 12 shows the standardised residuals of the ANOVA models as a function of the experimental order, the colours refer to the used tool. It should be noted that it not possible to observe any trend that is function of the experimental order, i.e. the tool wear evolution did not apparently influence 
the analysis. This was confirmed by the manual inspection of the tools before and after the entire cutting procedures.

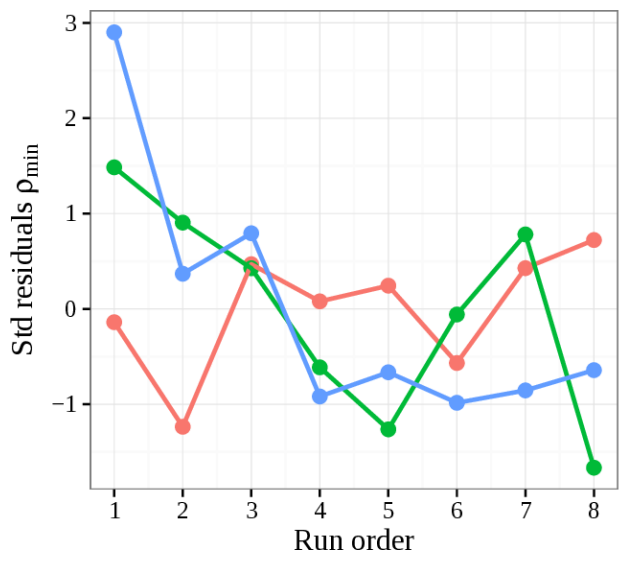

(a) $\rho_{\min }$

$\mathrm{h}$

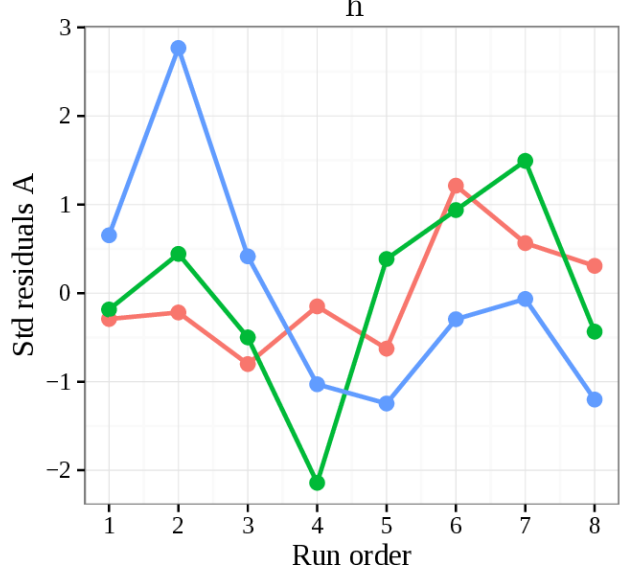

(c) $A$

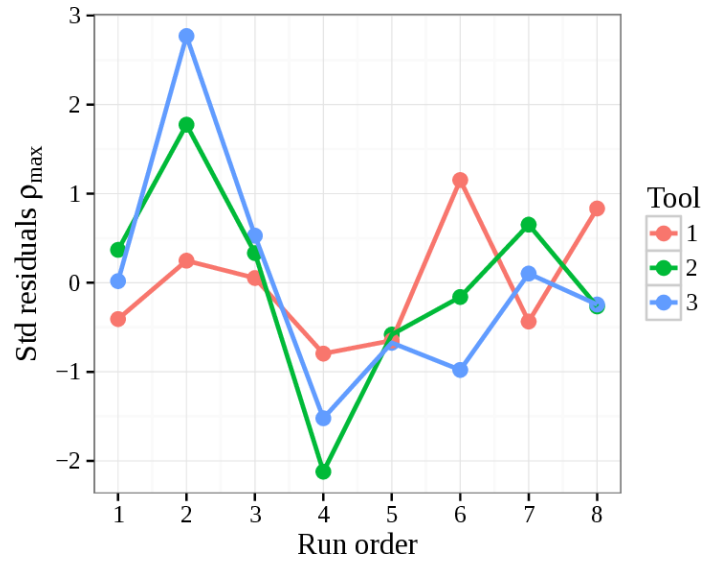

(b) $\rho_{\max }$

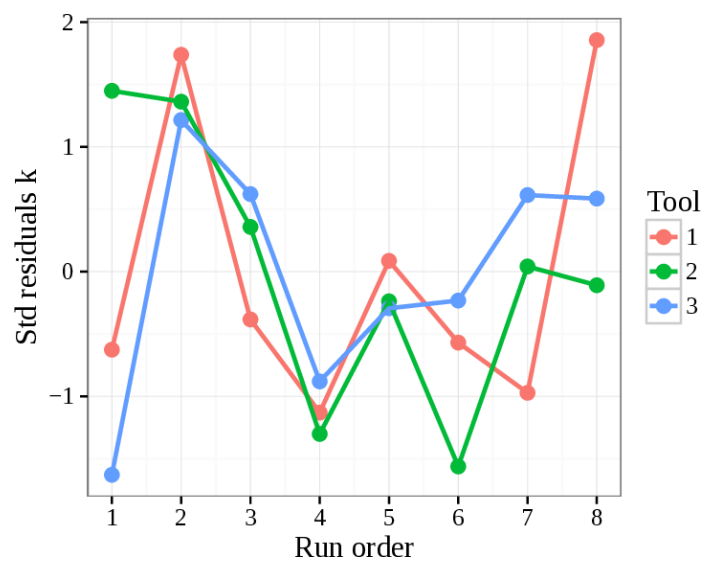

(d) $k$

Figure 12: Standardised residuals vs run order

\section{Sensitivity Analysis on the Imaging Algorithms}

In this Section, a sensitivity concerning the resolution necessary to compute the parameters of interest is performed. A sub sampling of the images, captured with the $20 \mathrm{x}$ lens, was first performed, then three holes were measured with different microscopes objectives. The objective of the sub sampling procedure is to check if is possible to use a image with less pixels to speed up the computation of the contour, while the scope of the second scenario is to verify if it is possible to use a different objective in order to reduce the measuring time. In both the analysed scenarios the parameters of the segmentation algorithm were not changed.

\subsection{Image resizing}

The measured images were resized in order to allow fast computational times and to reduce the storage space. The operation was performed in MATLAB ${ }^{\circledR}$ using a bi-cubic interpolation, two resize factors were analysed: 0.5 and 0.25 that correspond to decimation factors of $50 \%$ and $75 \%$ respectively. Figure 13 depicts the segmentation of an hole using the sub-sampled images along with the original image. The segmented holes appear qualitatively similar. A magnification of the extracted radii, in polar coordinates, is shown in Figure 14. Increasing the decimation, the segmented profiles are smoother with the highest difference located on peaks and valleys of the profile. 


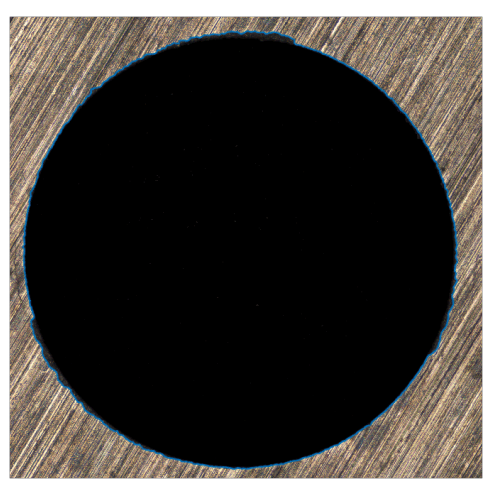

(a) $0 \%$ decimation

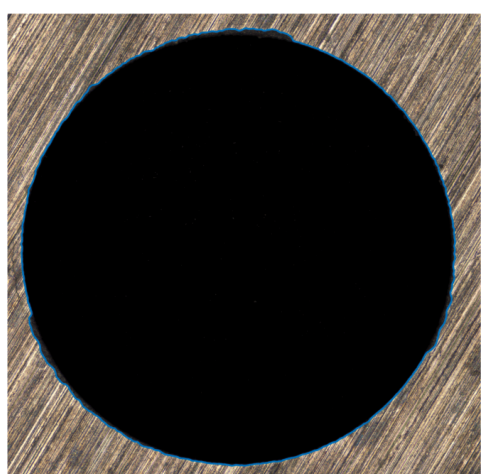

(b) $50 \%$ decimation

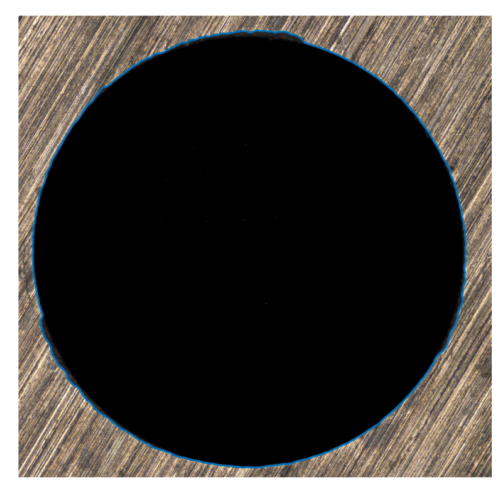

(c) $75 \%$ decimation

Figure 13: Hole segmentation after sub-sampling the image

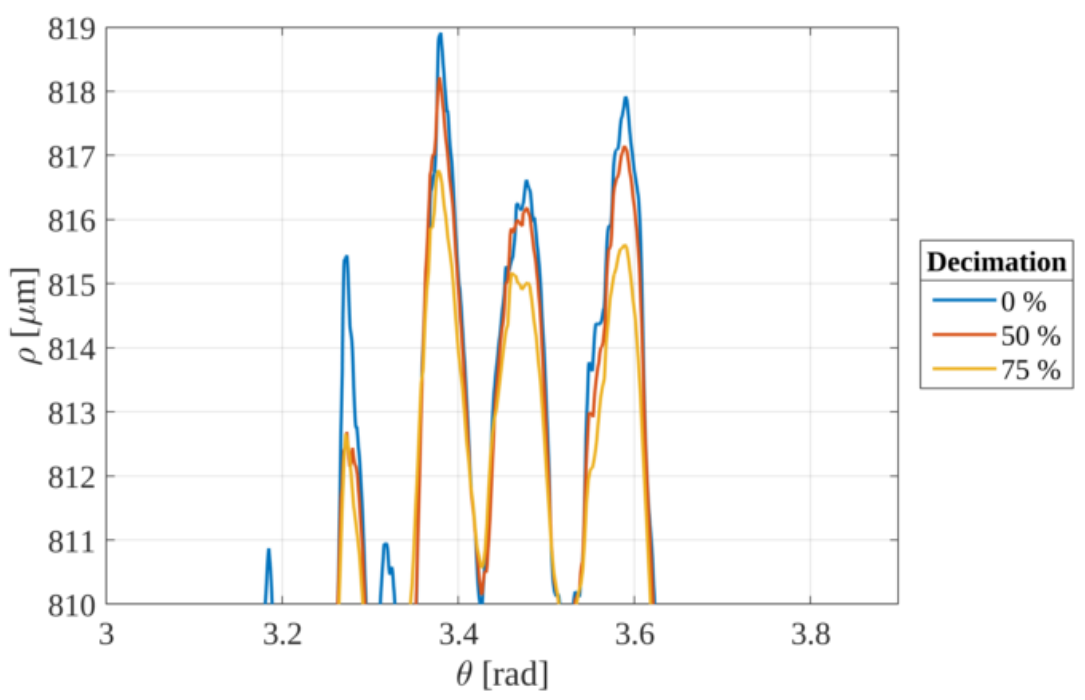

Figure 14: Magnification of the estimated radii as a function of the decimation

The quality indices reported in Table 2 were extracted for all the manufactured parts. The values of the differences, compared with the measured image, along with the mean and the $95 \%$ confidence interval, are shown in Figure 15. A paired t-test was performed to check whether any difference in the $\rho_{\min }, \rho_{\max }, A$ or $k$ exist. The p-values and the estimated difference on the mean are reported in Table 3 ; the average differences on the estimation of $\rho_{\min }$ and $\rho_{\max }$ were below $1 \%$, while the percentage error on the estimation of the defect area are $-3.17 \%$ and $-4.91 \%$ for the $50 \%$ and $75 \%$ decimation, respectively. On the other hand, the percentage maximum differences on the area estimation were $-10.48 \%$ and $-15.10 \%$; this high difference can be due to the segmentation algorithm, although it achieves a sub-pixel resolution, the accuracy of the method decreases as the decimation percentage increases. Due to the smoother profiles extracted after the sub-sampling step, the average differences on the kurtosis of the DFT amplitude were $-47.30 \%$ and $-71.52 \%$.

If the index of interest is one between $\rho_{\min }, \rho_{\max }$ or the range $\left(\rho_{\max }-\rho_{\min }\right)$ the accuracy lost on the estimation of these values is negligible, while there may be high error on the estimation on the defect area and on the kurtosis. 


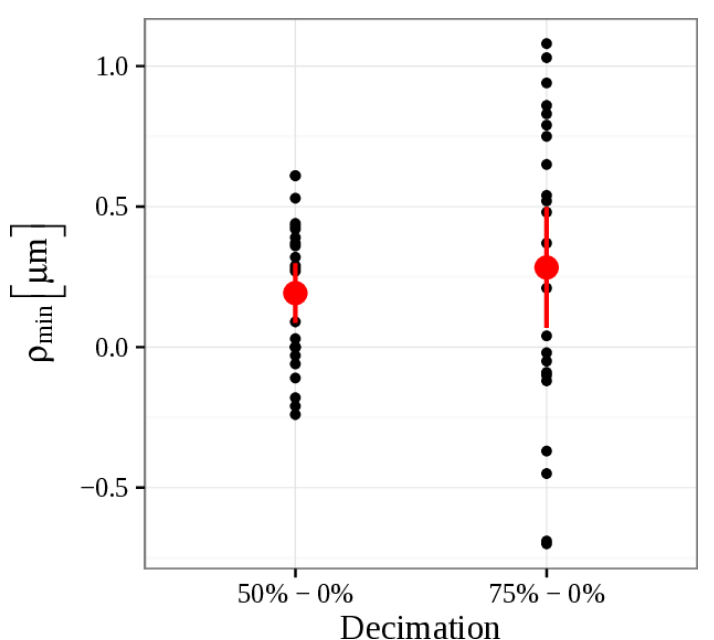

(a) $\rho_{\text {min }}$

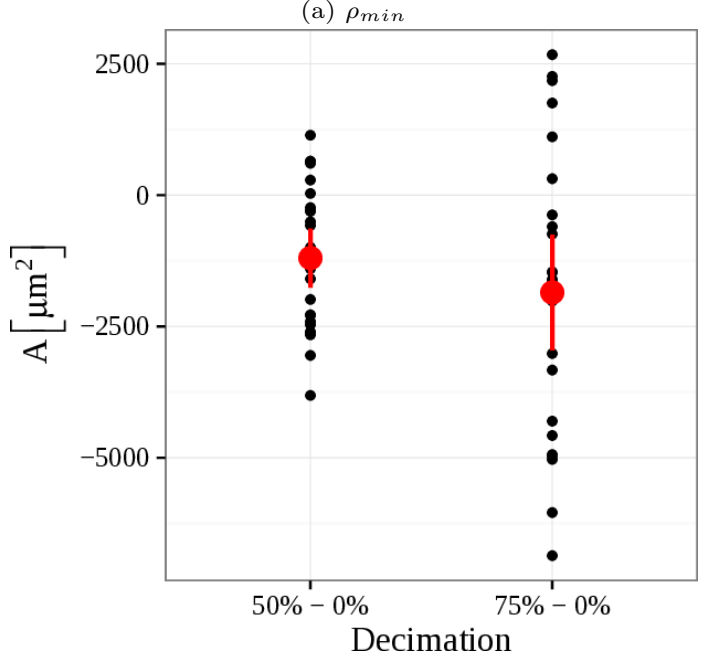

(c) $A$

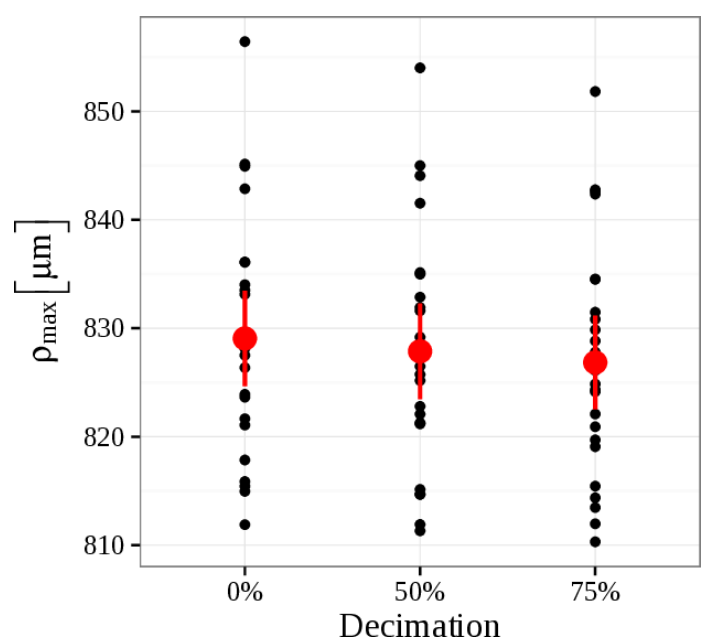

(b) $\rho_{\max }$

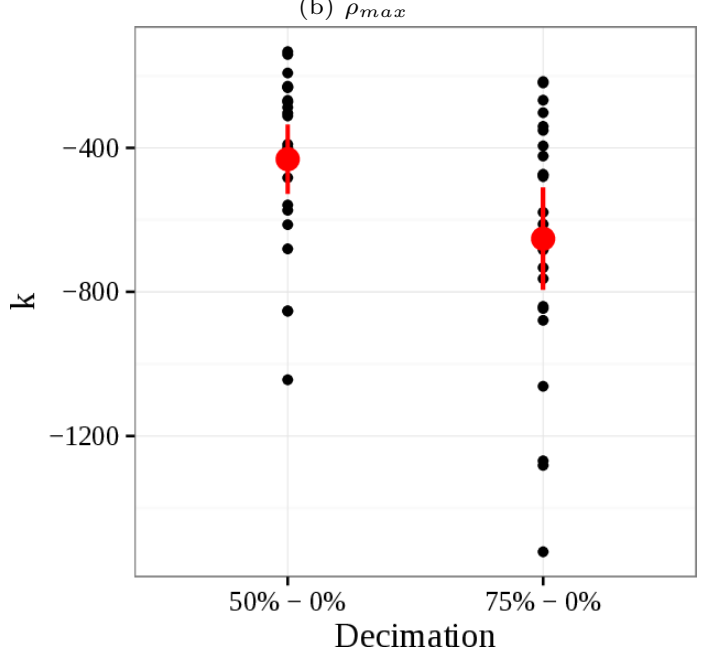

(d) $k$

Figure 15: Differences of computed indices and $95 \%$ confidence intervals

\begin{tabular}{l|r|r|r|r|r|r}
\hline \multirow{2}{*}{ Index } & \multicolumn{3}{|c|}{$50 \%-0 \%$} & \multicolumn{3}{|c}{$75 \%-0 \%$} \\
\cline { 2 - 7 } & P-value & Diff (\%) & Max diff (\%) & P-value & Diff (\%) & Max diff (\%) \\
\hline$\rho_{\min }$ & $<0.01$ & 0.02 & 0.08 & $<0.01$ & 0.04 & 0.14 \\
$\rho_{\max }$ & $<0.01$ & -0.14 & -0.49 & $<0.01$ & -0.27 & -0.68 \\
$A$ & $<0.01$ & -3.17 & -10.48 & $<0.01$ & -4.91 & -15.10 \\
$k$ & $<0.01$ & -47.30 & -74.55 & $<0.01$ & -71.52 & -82.60 \\
\hline
\end{tabular}

Table 3: Comparison between full resolution images and sub-sampled

\subsection{Effect of different microscope objectives}

To evaluate the accuracy of the measurement with lower magnification - i.e. lower measuring time, a total of three holes were measured both with the $5 \mathrm{x}, 10 \mathrm{x}$ and $20 \mathrm{x}$ objectives. The holes were selected based on the quality of the hole border: good (\#7), medium (\#16) and bad (\#19). The algorithm described in Section 4 was then applied to the holes measured with the $5 \mathrm{x}$ and $10 \mathrm{x}$ objectives. An hole measured with the different objectives with the segmented contours superimposed is shown in Figure 16. Table 4 reports the values of the quality indices of interest. It is possible to derive the same conclusion of the sub-sampled images: if the index of interest is a dimensional value - i.e. $\rho_{\min }$, $\rho_{\max }$ or the range, it is possible to measure the sample with a smaller magnification objective, whilst if the quality of interest is some more complex indicator - such as the defect area or the kurtosis - a bigger magnification objective is preferable. 


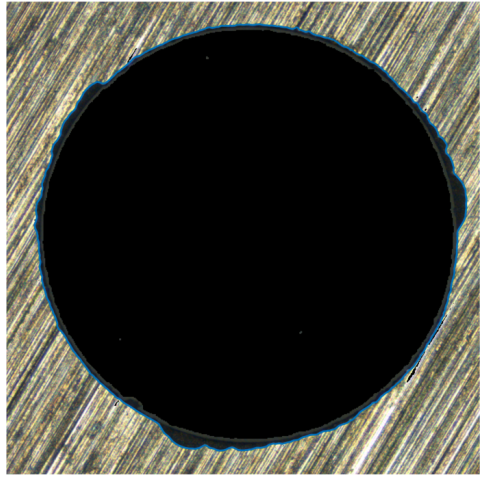

(a) $5 \mathrm{x}$

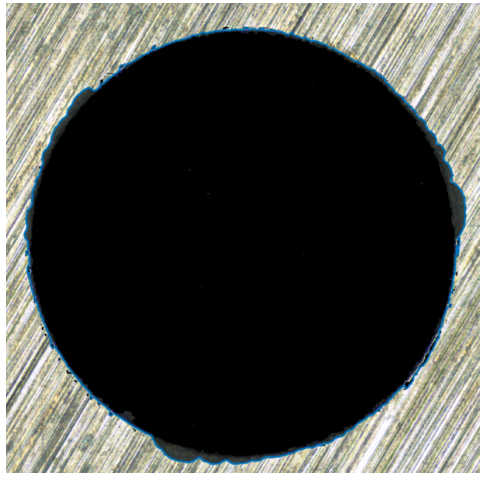

(b) $10 \mathrm{x}$

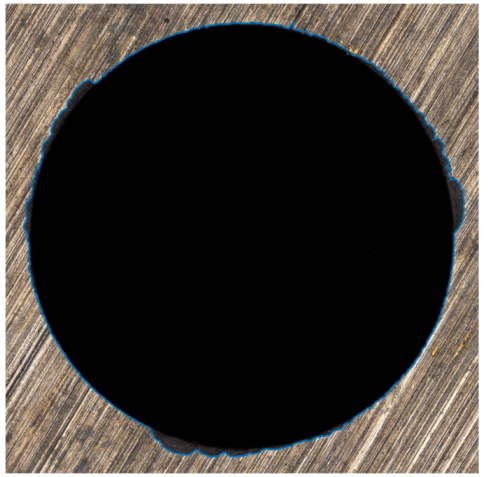

(c) $20 \mathrm{x}$

Figure 16: Acquisition and hole segmentation with different objectives (hole \# 19)

\begin{tabular}{l|c|c|c|c|c|c|c|c|c|c|c|c}
\hline \multirow{2}{*}{ Hole } & \multicolumn{3}{|c|}{$\rho_{\min }(\mu \mathrm{m})$} & \multicolumn{3}{c|}{$\rho_{\max }(\mu \mathrm{m})$} & \multicolumn{3}{c}{$A\left(\mu^{2}\right)$} & \multicolumn{2}{c}{$k$} \\
\cline { 2 - 12 } & $5 \mathrm{x}$ & $10 \mathrm{x}$ & $20 \mathrm{x}$ & $5 \mathrm{x}$ & $10 \mathrm{x}$ & $20 \mathrm{x}$ & $5 \mathrm{x}$ & $10 \mathrm{x}$ & $20 \mathrm{x}$ & $5 \mathrm{x}$ & $10 \mathrm{x}$ & $20 \mathrm{x}$ \\
\hline 7 & 799.12 & 800.20 & 800.51 & 825.00 & 826.19 & 827.51 & 32895 & 29382 & 26840 & 216.77 & 226.24 & 635.83 \\
16 & 799.26 & 799.84 & 799.79 & 818.76 & 820.54 & 821.07 & 28008 & 35840 & 31664 & 105.87 & 186.89 & 825.70 \\
19 & 799.53 & 800.68 & 800.91 & 853.26 & 854.62 & 856.43 & 71811 & 69965 & 65591 & 340.14 & 489.40 & 1135.16 \\
\hline
\end{tabular}

Table 4: Indices computed using different objectives

\section{Conclusions}

This study implemented an automatic inspection algorithm of small drilled holes edges, obtained on sintered tungsten carbide by diamond coated drilling tools.

The proposed image processing methodology was capable of automatically extract the holes' profiles and allows the characterisation of chipping defects (with amplitudes in the range of 10-50 $\mathrm{mm}$ ) on this innovative manufacturing method.

The algorithm was tested on multiple real test cases showing reliable and robust results. The automatic inspection produced equivalent results in respect to border extraction procedures carried out manually on the same microscopes analysis, demonstrating the high precision of the method. Together with the showed sub-pixel accuracy and the identification speed, this enables cutting process monitoring, process optimisation and in-line inspection procedures implementations.

The sensitivity analysis showed that if the parameters of interest are extreme values, i.e. $\rho_{\min }, \rho_{\max }$ or the range of the defect, a sub-sampling of the image can be performed to allow lower measurement and processing times. On the contrary, if the quality index of interest is the defect area the full resolution image has to by analysed.

A parameter able to characterise the profiles affected by lobes was proposed consisting in the kurtosis of the spatial spectrum amplitude.

The present study shows that the chipping defect extension and lobing tendency, cannot be explained by the tested cutting parameters. In this regards, further studies will be devoted to deepen the chipping mechanism analysis and to test this automatic inspection procedure in real production environment.

\section{Acknowledgement}

The authors want to thank F.I.L.M.S. Spa for providing the material and the tools used in this study and S. Crespi for supporting the experimental phase. LP acknowledges the UK's Engineering and Physical Sciences Research Council (EPSRC) funding of the grant EP/R024162/1.

\section{References}

[1] M. Okada, A. Yoshida, T. Furumoto, H. Watanabe, N. Asakawa, M. Otsu, Mechanisms and characteristics of direct cutting of tungsten carbide using a diamond-coated carbide end mill, The International Journal of Advanced Manufacturing Technology 86 (2016) 1827-1839. 
[2] K. Giasin, S. Ayvar-Soberanis, T. French, V. Phadnis, 3D Finite Element Modelling of Cutting Forces in Drilling Fibre Metal Laminates and Experimental Hole Quality Analysis, Applied Composite Materials 24 (2017) 113-137.

[3] A. Faraz, D. Biermann, K. Weinert, Cutting edge rounding: An innovative tool wear criterion in drilling CFRP composite laminates, International Journal of Machine Tools and Manufacture 49 (2009) 1185-1196.

[4] A. D’Orazio, M. El Mehtedi, A. Forcellese, A. Nardinocchi, M. Simoncini, Tool wear and hole quality in drilling of CFRP/AA7075 stacks with DLC and nanocomposite TiAlN coated tools, Journal of Manufacturing Processes 30 (2017) 582-592.

[5] E. Aoyama, H. Nobe, T. Hirogaki, Drilled hole damage of small diameter drilling in printed wiring board, Journal of Materials Processing Tech. 118 (2001) 436-441.

[6] G. Beruvides, R. Quiza, R. del Toro, F. Castaño, R. E. Haber, Correlation of the holes quality with the force signals in a microdrilling process of a sintered tungsten-copper alloy, International Journal of Precision Engineering and Manufacturing 15 (2014) 1801-1808.

[7] D. Lv, Y. Zhang, Y. Peng, High-frequency vibration effects on hole entrance chipping in rotary ultrasonic drilling of BK7 glass, Ultrasonics 72 (2016) 47-56.

[8] J. Wang, H. Zha, P. Feng, J. Zhang, On the mechanism of edge chipping reduction in rotary ultrasonic drilling: A novel experimental method, Precision Engineering 44 (2015) 231-235.

[9] V. De Albuquerque, J. Tavares, L. Durao, Evaluation of Delamination Damage on Composite Plates using an Artificial Neural Network for the Radiographic Image Analysis, Journal of Composite Materials 44 (2010) 1139-1159.

[10] J. Canny, A computational approach to edge detection, IEEE Transactions on Pattern Analysis and Machine Intelligence PAMI-8 (1986) 679-698.

[11] V. Caselles, R. Kimmel, G. Sapiro, Geodesic active contours, International Journal of Computer Vision 22 (1997) 61-79.

[12] T. Chan, L. Vese, Active contours without edges, IEEE Transactions on Image Processing 10 (2001) 266-277.

[13] H. Watanabe, H. Tsuzaka, M. Masuda, Microdrilling for printed circuit boards ( PCBs )Influence of radial run-out of microdrills on hole quality, Precision Engineering 32 (2008) 329335 .

[14] T. Birdal, Maximum inscribed circle using distance transform, 2011.

[15] S. Osher, J. A. Sethian, Fronts propagating with curvature-dependent speed: Algorithms based on hamilton-jacobi formulations, Journal of Computational Physics 79 (1988) 12-49.

[16] A. Faraz, D. Biermann, K. Weinert, Cutting edge rounding: An innovative tool wear criterion in drilling CFRP composite laminates, International Journal of Machine Tools and Manufacture 49 (2009) 1185-1196.

[17] J. P. Davim, J. C. Rubio, A. M. Abrao, A novel approach based on digital image analysis to evaluate the delamination factor after drilling composite laminates, Composites Science and Technology 67 (2007) 1939-1945. 